Gut and Liver, Vol. 11, No. 2, March 2017, pp. 243-252

\title{
Açaí Berries Inhibit Colon Tumorigenesis in Azoxymethane/Dextran Sulfate Sodium-Treated Mice
}

\author{
Yoon Jin Choi ${ }^{1}$, Yoon Jeong Choi ${ }^{1}$, Nayoung $\mathrm{Kim}^{1,2}$, Ryoung Hee Nam ${ }^{1}$, Seonmin Lee ${ }^{1}$, Hye Seung Lee ${ }^{3}$, Ha-Na Lee ${ }^{4}$,
} Young-Joon Surh ${ }^{4}$, and Dong Ho Lee ${ }^{1,2}$

${ }^{1}$ Department of Internal Medicine, Seoul National University Bundang Hospital, Seongnam, ${ }^{2}$ Department of Internal Medicine and Liver Research Institute, Seoul National University College of Medicine, Seoul, ${ }^{3}$ Department of Pathology, Seoul National University Bundang Hospital, Seongnam, and ${ }^{4}$ Tumor Microenvironment Global Core Research Center, Seoul National University College of Pharmacy, Seoul, Korea

Background/Aims: The aim of this study was to investigate the protective effect of açaí against azoxymethane (AOM)/ dextran sulfate sodium (DSS)-induced colorectal cancer development. Methods: The effect of açaí on tumorigenesis was assessed by evaluating tumor incidence, multiplicity and invasiveness in the mouse colon. The levels of myeloperoxidase (MPO) and proinflammatory cytokines (tumor necrosis factor $\alpha$ [TNF- $\alpha$ ], interleukin [IL]-1 $\beta$, and IL-6) were measured via enzyme-linked immunosorbent assay. Protein levels of cyclooxygenase 2 (COX-2), proliferating cell nuclear antigen (PCNA), B-cell lymphoma 2 (Bcl-2), Bcl-2-associated death promoter (Bad) and cleaved-caspase-3 were assessed by immunoblotting. Results: Administration of pellets containing $5 \%$ açaí powder reduced the incidences of both colonic adenoma and cancer (adenoma, $23.1 \%$ vs $76.9 \%$, respectively, $p=0.006$; cancer, $15.4 \%$ vs $76.9 \%$, respectively, $p=0.002$ ). In the açaí-treated mice, the MPO, TNF- $\alpha$, IL-1 $\beta$ and IL-6 levels in the colon were significantly down-regulated. Açaí inhibited PCNA and Bcl-2 expression and increased Bad and cleaved-caspase-3 expression. In vitro studies demonstrated that açaí treatment reduced lipopolysaccharide-induced expression of TNF- $\alpha$, IL-1 $\beta$, IL- 6 and COX-2 in murine macrophage RAW 264.7 cells. Conclusions: Açaí demonstrated protective effects against AOM/DSS-induced colon carcinogenesis, which suggests that the intake of açaí may be beneficial for the prevention of human colon cancer. (Gut Liver 2017;11:243-252)

Key Words: Açaí berry; Colorectal neoplasms; Anti-inflammatory; Proapoptotic

\section{INTRODUCTION}

Colorectal cancer (CRC) is the third most common cancer worldwide and the fourth most frequent cause of cancer-related death. ${ }^{1}$ Chronic inflammation is a risk factor for tumorigenesis, and epidemiological data suggest that up to 15\% of human cancers are associated with inflammation. ${ }^{2,3}$ Colitis-associated cancer (CAC) is a type of colon cancer preceded by clinically detectable inflammatory bowel disease, such as Crohn's disease or ulcerative colitis (UC). ${ }^{4}$ Indeed, UC has been reported to increase the cumulative risk of CAC by up to $18 \%$ to $20 \%{ }^{5}$

Accumulating evidence suggests that chronic inflammatory conditions predispose cells to malignant transformation, thereby promoting tumorigenesis. In the inflammatory site, activated inflammatory cells produce reactive oxygen species (ROS) and reactive nitrogen intermediates, the persistent formation of which can induce DNA damage and mutation in surrounding cells. ${ }^{6}$ Moreover, ROS produced by immune cells can stimulate epithelial cells to induce sustained production of intracellular ROS, leading to severe mutations or epigenetic silencing of tumor suppressor genes. ${ }^{7}$ As well as oxidative stress, chronic exposure to proinflammatory cytokines, such as interleukin (IL)-6, IL-1 $\beta$, and tumor necrosis factor $\alpha$ (TNF- $\alpha$ ), causes tumorigenesis. ${ }^{8-11}$

As chronic inflammation is a risk factor for colon tumorigenesis, nonsteroidal anti-inflammatory drugs have been considered to be effective chemopreventive agents for CRC. However, their long-term administration results in gastrointestinal side effects; therefore, alternative therapeutic approaches are needed to manage or prevent inflammation-associated CRC. ${ }^{12}$ In this regard, fruits and vegetables containing various compounds with antioxidant and anti-inflammatory properties have been con-

Correspondence to: Nayoung Kim

Department of Internal Medicine, Seoul National University Bundang Hospital, 82 Gumi-ro 173beon-gil, Bundang-gu, Seongnam 13620, Korea

Tel: +82-31-787-7008, Fax: +82-31-787-4051, E-mail: nayoungkim49@empas.com

Received on February 3, 2016. Revised on May 25, 2016. Accepted on June 28, 2016. Published online December 16,2016

pISSN 1976-2283 eISSN 2005-1212 https://doi.org/10.5009/gnl16068

Yoon Jin Choi and Yoon Jeong Choi contributed equally to this work as first authors.

@ This is an Open Access article distributed under the terms of the Creative Commons Attribution Non-Commercial License (http://creativecommons.org/licenses/by-nc/4.0) which permits unrestricted non-commercial use, distribution, and reproduction in any medium, provided the original work is properly cited. 
sidered promising sources of chemopreventive agents for CRC due to their low toxicity. ${ }^{13}$ The açai berry has attracted much attention in this regard. The açai species Euterpe oleracea Mart. is an exotic fruit that contains high levels of polyphenols, especially anthocyanin and proanthocyanidin (mainly cyanidin 3-0glucoside and cyanidin 3-0-rutinoside). ${ }^{14}$ Dietary administration of açai attenuated atherosclerosis in apolipoprotein-E-deficient mice, and cigarette smoke-induced lung inflammation through its anti-inflammatory and antioxidant activities. ${ }^{15,16}$ Açaí also exerts anticancer effects by promoting apoptosis of cancer cells (e.g., human SW-480 colon cancer cells and human leukemic-60 cells). ${ }^{17,18}$ Furthermore, açaí inhibits dimethylhydrazineinduced colon carcinogenesis and N-nitrosomethylbenzylamineinduced esophageal cancer development in rats. ${ }^{19,20}$

Although anticancer properties of açai have been suggested, the protective effect of açai on inflammation-associated CRC has not been investigated to date. This prompted us to investigate the chemopreventive effect of dried açai powder on azoxymethane (AOM)/dextran sulfate sodium (DSS)-induced colorectal adenoma and cancer in mice, with a focus on its antiinflammatory, proapoptotic and antioxidant properties.

\section{MATERIALS AND METHODS}

\section{Chemicals}

Açaí berries were collected in Belem, Brazil, and spray-dried using an industrial spray-dryer system with maltodextrin DE10 as a carrier agent. ${ }^{21}$ Açaí pulp powder was produced by Centroflora Group Brazil (Botucatu, Brazil) with the following characteristics: moisture 6\%, volumetric density 350 to $650 \mathrm{~g} / \mathrm{L}$, and total polyphenol content $0.5 \% .{ }^{21}$ Freeze-dried açaí pulp powder was purchased through Boto Superfood Co., Ltd. (Seoul, Korea) which imported the end product. Freeze-dried açai powder was stored at $-20^{\circ} \mathrm{C}$ until analyzed. A cereal-based commercial diet for mice containing 2.5\% and 5\% açaí powder was specially formulated by the Orient Bio Group (Seongnam, Korea) by a natural drying method according to the National Research Council's recommendation to meet rodent nutritional needs. ${ }^{21}$

For açaí treatment in vitro, açaí powder $(0.5 \mathrm{~g})$ was freshly dissolved in $5 \mathrm{~mL}$ of phosphate-buffered saline, $\mathrm{pH}$ 7.4. This mixture was vortexed repeatedly and allowed to sit at room temperature for 2 hours. Prior to use, insoluble particles were removed by centrifugation and subsequent filtration using a 0.22 $\mu \mathrm{m}$ cellulose-acetate syringe filter.

\section{Animal experiments}

All experimental procedures were approved by the Institutional Animal Care and Use Committee of Seoul National University Bundang Hospital (BA1310-139/091-01) on 21 October 2013. The procedure was in accordance with the ARRIVE (Animals in Research: Reporting In Vivo Experiments) statement. Male ICR mice (4 weeks of age) were purchased from Orient Co., Ltd. (Seoul, Korea) and housed in a cage maintained at $23^{\circ} \mathrm{C}$, with a 12/12 hour light/dark cycle under specific pathogen-free conditions. Experimental groups included group 1 (untreated control, $n=8)$; group $2(n=13$, treated with AOM and DSS); group 3 to 4 ( $n=13$ per group, were treated AOM/DSS and açaí [2.5\% for group 3 and 5\% for group 4]); group $5(n=8)$ was treated with only açai (5\%) (Fig. 1A). Mice in groups 2 to 4 were given a single intraperitoneal injection of $10 \mathrm{mg} / \mathrm{kg}$ AOM (Sig-
A

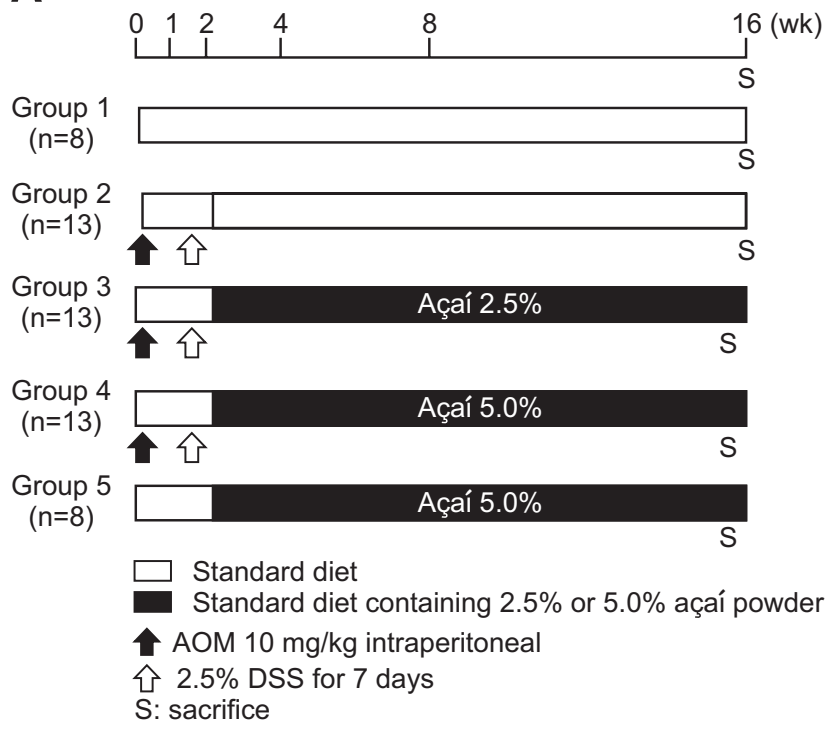

B

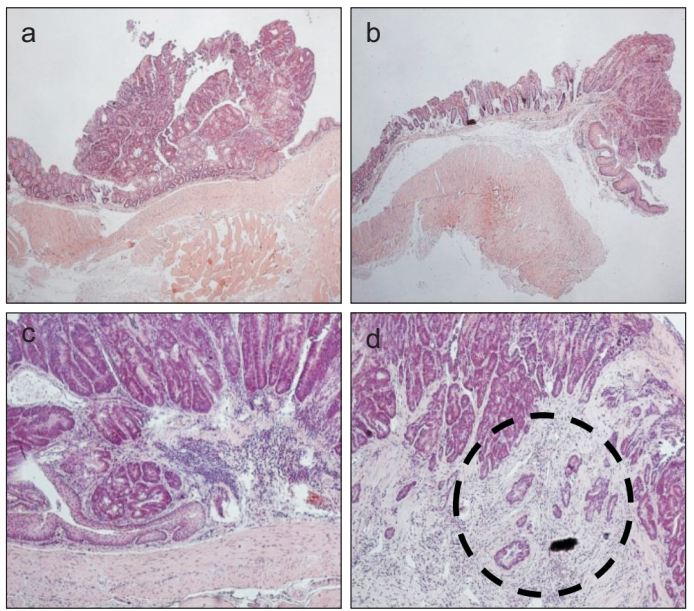

Fig. 1. Azoxymethane (AOM)/dextran sulfate sodium (DSS)-induced mouse colon carcinogenesis model. (A) Total experimental protocols. (B) Representative histological images of (a) adenoma, (b) cancer, (c) mucosal cancer, and (d) submucosal invasion of cancer (H\&E stain; a, b, ×40; c, d, $\times 100$ ). Cancer cells were identified in the submucosa (circle). 
ma-Aldrich, St. Louis, MO, USA). For induction of colitis, DSS (MP Biomedicals, Aurora, OH, USA) was prepared in drinking water at a concentration of $2.5 \%(\mathrm{w} / \mathrm{v}) .^{22}$ Starting 1 week after AOM injection, mice received 2.5\% DSS in drinking water for 7 days. Subsequently, groups 3 to 4 received 2.5\% and 5\% açaícontaining diets for 14 weeks, respectively. All animals were euthanized at 16 weeks.

\section{Gross and histopathological evaluation of colonic mucosa}

Complete autopsies were performed and the colons from the cecum to rectum were immediately removed, flushed with phosphate buffered saline, and opened longitudinally. Polypoid lesions were counted in the whole colon by three gastroenterologists in a blinded manner and tumor multiplicity was defined as the number of gross polyps approved by all of the three gastroenterologists.

The rectum (up to $3 \mathrm{~cm}$ from the anal verge) and other segments including any grossly proven polyps larger than $2 \mathrm{~mm}$ in diameter were fixed in phosphate-buffered formalin and stained with hematoxylin and eosin for histopathological examination. Another portion was flash-frozen in lipid nitrogen and kept at $-70^{\circ} \mathrm{C}$ for enzyme-linked immunosorbent assay (ELISA), Western blot and polymerase chain reaction (PCR) analyses. The tumors were classified as adenomas or adenocarcinomas according to Hamilton and Aaltonen ${ }^{23}$ (Fig. 1B). In addition, the depth of invasion by colonic adenocarcinomas was described as mucosa and "into the submucosa and muscularis" (Fig. 1B) and their incidence (percentage of rats with tumor) was assessed.

\section{Cytokine measurement}

An ELISA was performed to measure cytokine levels using the appropriate kits from R\&D systems (Minneapolis, MN, USA). All assays were performed in triplicate, and data are shown as means \pm standard error (SE).

\section{Western blot analysis}

Protein extracts were isolated using RIPA buffer (Cell Signaling Technology, Beverly, MA, USA). Protein samples were mixed with an equal volume of $5 \times$ SDS sample buffer, boiled for 5 minutes, and then separated in $8 \%$ to 12\% SDS-PAGE gels. After electrophoresis, proteins were transferred to polyvinylidene difluoride membranes. The membranes were blocked with 5\% nonfat dry milk in Tris-buffered saline with Tween-20 buffer (TBS-T) for 1 hour at room temperature. Membranes were incubated overnight at $4^{\circ} \mathrm{C}$ with specific antibodies. Primary antibodies were removed by washing the membranes three times in TBS-T, and incubated for 2 hours with horseradish peroxidase-conjugated antirabbit or antimouse immunoglobulin (Santa Cruz Biotechnology, Dallas, TX, USA). Following three washes with TBS-T, antigen-antibody complexes were detected using the SuperSignal West Pico Chemiluminescence System (Thermo Fisher Scientific, Rockford, IL, USA). The incubation condi- tions were as follows: anti-cyclooxygenase2 (COX-2) antibody (1:1,000; Cayman Chemical, Ann Arbor, MI, USA), anti-proliferating cell nuclear antigen (PCNA) antibody (1:1,000; Santa Cruz Biotechnology), anti-B-cell lymphoma 2 (Bcl-2) antibody (1:1,000; Santa Cruz Biotechnology), anti-Bcl-2-associated death promoter (Bad) antibody (1:1,000; Santa Cruz Biotechnology), anti-cleaved caspase 3 antibody (1:1,000; Cell Signaling Technology), anti-heme oxygenase 1 (HO-1) antibody (1:1,000; Abcam Inc., Cambridge, UK) or anti-NQO 1 [NAD(P)H:quinone oxidoreductase 1] antibody (1:1,000; Abcam Inc.).

\section{Quantitative real-time PCR analysis}

Total RNA from was isolated using RNeasy Plus Mini Kits (Qiagen, Valencia, CA, USA). Reverse transcription was performed using the High Capacity cDNA Reverse Transcription Kit (Applied Biosystems, Foster City, CA, USA). Real-time qPCR for mRNA expression was performed using SYBR Green probes and an ABI 7500 instrument. The mRNA expression of all genes was normalized to that of GAPDH. The primer sequences were as follows: TNF- $\alpha, 5^{\prime}$-TCT CAT GCA CCA CCA TCA AGG ACT3'and 5'-ACC ACT CTC CCT TTG CAG AAC TCA-3'; IL-1 $\beta$, 5'ACT CAT TGT GGC TGT GGA GA- $3^{\prime}$ and $5^{\prime}$-TTG TTC ATC TCG GAG CCT GT-3'; COX-2, 5'-TGC CTG GTC TGA TGA TGT ATG CCA-3' and 5'-AGT AGT CGC ACA CTC TGT TGT GCT-3'; GAPDH, 5'-TGA AGC AGG CAT CTG AGG G-3' and 5'-CGA AGG TGG AAG AGT GGG AG-3' (forward and reverse, respectively).

\section{Cell culture}

Mouse RAW 264.7 macrophages were obtained from the American Type Culture Collection (Manassas, VA, USA) and maintained in Dulbecco's Modified Eagle's medium (DMEM) containing 10\% fetal bovine serum (FBS), $100 \mathrm{U} / \mathrm{mL}$ penicillin and $100 \mu \mathrm{g} / \mathrm{mL}$ streptomycin (Gibco BRL, San Francisco, CA, USA) at $37^{\circ} \mathrm{C}$ in a humidified 5\% $\mathrm{CO}_{2}$ atmosphere. RAW 264.7 cells were treated with lipopolysaccharide (LPS) $(200 \mathrm{ng} / \mathrm{mL}$; Sigma Aldrich) in the presence or absence of açai $(20,40,80$, or $100 \mu \mathrm{g} / \mathrm{mL}$ ). Human colonic epithelial cells (CCD841CoN) were kindly provided by Y.J.S. (Seoul National University, Korea) and were maintained in DMEM containing 10\% FBS, $100 \mathrm{U} / \mathrm{mL}$ penicillin and $100 \mu \mathrm{g} / \mathrm{mL}$ streptomycin. ${ }^{22}$

\section{Statistical analysis}

Data are expressed as means \pm SE. Statistical analyses were conducted using the GraphPad Prism (GraphPad Software Inc., La Jolla, CA, USA) and SPSS version 12.0 (SPSS Inc., Chicago, IL, USA) software. Statistical significance was determined using the Mann-Whitney $U$ test and $\mathrm{p}<0.05$ was considered to indicate a statistically significant difference. 


\section{RESULTS}

\section{Açaí attenuates AOM/DSS-induced colon carcinogenesis}

To investigate whether açaí has preventive effects on inflammation-induced carcinogenesis, we used the AOM-initiated and DSS-promoted mouse CRC model. After 2.5\% DSS administra- tion, mice were fed either a normal or açaí-containing diet for 14 weeks (Fig. 1). At week 16, nodular colonic adenomas were macroscopically found in the middle and distal colon of mice treated with AOM/DSS (Fig. 2A). Administration of 5\% açai reduced the incidence of both colonic adenoma and cancer (adenoma, 23.1\% vs 76.9\%, p=0.006; cancer, 15.4\% vs 76.9\%,
A

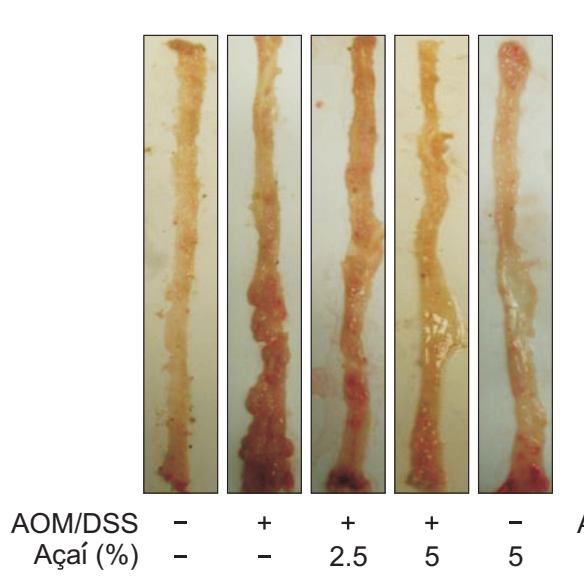

B

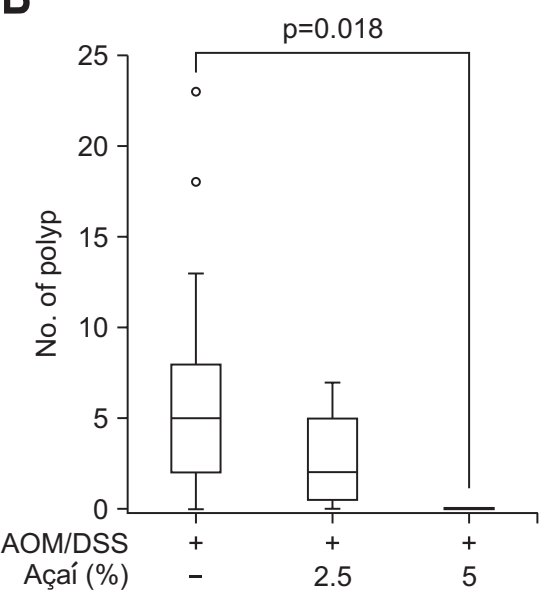

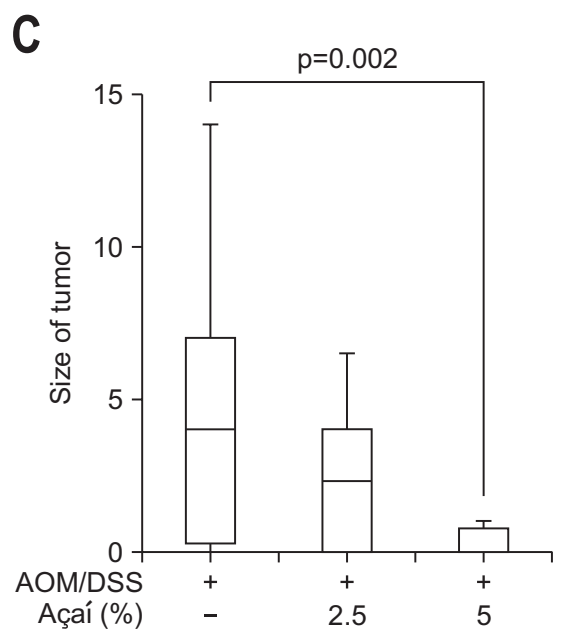

D
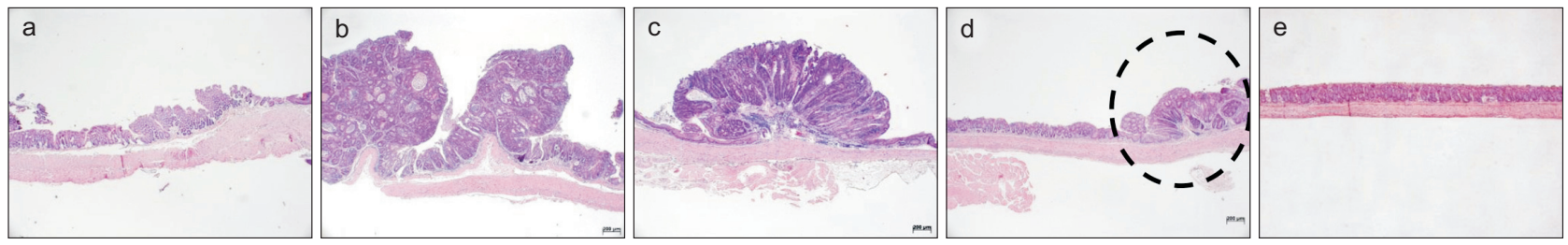

Fig. 2. Açaí inhibits azoxymethane (AOM)/dextran sulfate sodium (DSS)-induced mouse colon carcinogenesis. (A) Representative macroscopic view of the mouse colon in each group. (B) More colonic tumors were identified in the distal colon and rectum of the AOM/DSS-only-treated group than in those of the AOM/DSS+açai 5\% group ( $\mathrm{p}=0.018$ ). (C) Larger colonic tumors were identified in the distal colon and rectum of the AOM/DSS-only-treated group than in those of the AOM/DSS+açai 5\% group ( $p=0.002)$. (D) Representative histological images (H\&E stain, $\times 40$, and scale bar, $200 \mu \mathrm{m}$ ) in (a) control, (b) AOM/DSS, (c) AOM/DSS+açaí 2.5\%, (d) AOM/DSS+açaí 5\% with the tumor portion indicated in a circle, and (e) açai 5\% alone. Animal treatments and other experimental conditions are described in the MATERIALS AND METHODS section.

Table 1. Incidence and Multiplicity of Colon Adenoma and Cancer

\begin{tabular}{|c|c|c|c|c|c|c|c|}
\hline Treatment group & No. & $\begin{array}{l}\text { Adenoma } \\
\text { incidence }\end{array}$ & $\begin{array}{l}\text { Cancer } \\
\text { incidence }\end{array}$ & $\begin{array}{l}\text { Adenoma/cancer } \\
\text { incidence }\end{array}$ & $\begin{array}{c}\text { Adenoma/cancer } \\
\text { multiplicity }\end{array}$ & $\begin{array}{c}\text { Size of } \\
\text { adenoma/tumor }\end{array}$ & Depth of invasion (n) \\
\hline Control & 8 & 0 & 0 & 0 & 0.00 & 0.00 & \\
\hline AOM/DSS & 13 & $76.9(10 / 13)$ & $76.9(10 / 13)$ & $76.9(10 / 13)$ & $6.62 \pm 2.01$ & $4.21 \pm 0.89$ & Mucosa (9), submucosa (1) \\
\hline AOM/DSS+açaí 2.5\% & 13 & $38.5(5 / 13)$ & $53.8(7 / 13)$ & $53.8(7 / 13)$ & $2.75 \pm 0.94$ & $2.29 \pm 0.69$ & Mucosa (6), submucosa (1) \\
\hline AOM/DSS+açaí 5\% & 13 & $23.1(3 / 13)$ & $15.4(2 / 13)$ & $23.1(3 / 13)$ & $0.85 \pm 0.53$ & $0.83 \pm 0.38$ & Mucosa (1), submucosa (1) \\
\hline Açaí 5\% & 8 & 0 & 0 & 0 & 0.00 & 0.00 & \\
\hline $\mathrm{p}$ trend & & $0.007^{*}$ & $0.002^{*}$ & $0.007^{*}$ & & & \\
\hline$p$-value ${ }^{\dagger}$ & & $0.018^{*}$ & $0.007^{*}$ & $0.022^{*}$ & $0.016^{*, S}$ & $0.002^{*}$ & \\
\hline p-value ${ }^{\ddagger}$ & & $0.006^{*}$ & $0.002^{*}$ & $0.006^{*}$ & $0.018^{*, \S}$ & $0.002^{* . \$}$ & \\
\hline p-value" & & 0.047 & 0.216 & 0.411 & 0.222 & 0.361 & \\
\hline
\end{tabular}

Data are presented as percent (number/total number) or mean \pm standard error.

AOM, azoxymethane; DSS, dextran sulfate sodium.

${ }^{*}$ Represents statistical significance; ${ }^{\dagger}$ Among three AOM/DSS-treated groups; ${ }^{\ddagger}$ Between AOM/DSS and AOM/DSS+açai 5\% groups; ${ }^{\S}$ Using one-way analysis of variance followed by a Scheffe test; "Between AOM/DSS and AOM/DSS+açaí 2.5\% groups. 
$\mathrm{p}=0.002$ ) (Table 1 ). Multiplicity of colonic adenoma or cancer was decreased in the $5 \%$ açai-fed mouse group compared to the AOM/DSS-only treated mouse group $(0.85 \pm 0.53$ vs $6.62 \pm 2.01$, $\mathrm{p}=0.018$ ) (Table 1, Fig. 2B). Administration of 5\% açaí reduced the tumor size $(0.83 \pm 0.38 \mathrm{~mm}$ vs $4.21 \pm 0.89 \mathrm{~mm})$ (Table 1, Fig. 2C). Histological examination also showed that AOM/DSS-induced CRCs or adenomas were alleviated by açaí administration in a dose-dependent manner (Fig. 2D). The groups fed 2.5\% açai did not show a significant reduction in the incidence or multiplicity of adenomas (Table 1).

Although all of the three AOM/DSS-treated groups had adenomas (Fig. 2D, b-d) and cancer with mucosal or submucosal invasion, even the group fed 5\% açai (Fig. 2D, d), the incidence and multiplicity of cancer were significantly decreased in the AOM/DSS-treated group with 5\% açaí.

\section{Açaí suppresses proinflammatory cytokine production, inhibits cell proliferation, and induces apoptosis}

Increased inflammatory cell influx and proinflammatory cytokine production is a hallmark of colorectal tumors. Therefore, we tested the effects of açai on myeloperoxidase (MP0) expression and proinflammatory cytokine production in the AOM/ DSS-induced CRC model. The expression levels of MPO and proinflammatory cytokines such as TNF- $\alpha$, IL- $1 \beta$ and IL- 6 were significantly increased in the AOM/DSS group, and açai administration reduced the proinflammatory cytokine levels (Fig. 3). Açaí administration also inhibited AOM/DSS-induced COX-2 expression in the mouse colon (Fig. 4A), the major proinflammatory enzymes whose expression is regulated by nuclear factor- $\kappa \mathrm{B}(\mathrm{NF}-\kappa \mathrm{B}){ }^{24}$ These results indicate that açai administration exerts an anti-inflammatory effect in the AOM/DSSinduced CRC model.

PCNA was evaluated as an important marker of cell pro- liferation in the colonic mucosa. ${ }^{25}$ As shown in Fig. 4A, the level of PCNA was significantly elevated in the colon of AOM/ DSS-treated mice, compared to control. However, in a dosedependent manner, açaí administration suppressed AOM/DSSinduced PCNA expression in the mouse colon, indicating that açaí inhibits cell proliferation.

Dysregulation of apoptosis plays a pivotal role in tumor progression and therapy resistance. ${ }^{18}$ We investigated the effect of açai on the apoptosis pathway in AOM/DSS-induced colon cancer progression. The protein levels of Bcl-2 were significantly inhibited by administration of 2.5\% and 5\% açaí, while 5\% açaí induced expression of Bad and cleaved-caspase- 3 in the mouse colon (Fig. 4B). This suggested that açaí triggers cell apoptosis by targeting the mitochondrial intrinsic proapoptotic pathway, which results in caspase- 3 cleavage.

\section{Açaí inhibits LPS-induced proinflammatory gene expression in RAW 264.7 cells}

Following evaluation of the anti-inflammatory activity of açai in the AOM/DSS-induced mouse colon cancer model, we examined its effect on cytokine expression in a mouse macrophage cell line (RAW 264.7) upon stimulation with LPS, which is one of the most potent proinflammatory stimuli for monocytes and macrophages. RAW 264.7 cells were pretreated with açai extract at concentrations ranging from 20 to $100 \mu \mathrm{g} / \mathrm{mL}$ for 1 hour and then stimulated with $200 \mathrm{ng} / \mathrm{mL}$ LPS for a 6 hours incubation period. As shown in Fig. 5, mRNA expression levels of TNF- $\alpha$, IL- $1 \beta$ and COX-2 were significantly increased by LPS stimulation (Fig. 5A-C). mRNA expressions of TNF- $\alpha$ and COX-2 in RAW 264.7 cells treated with LPS were significantly reduced by 40 and $80 \mu \mathrm{g} / \mathrm{mL}$ of açaí extract compared with only LPS-treated macrophages, while that of IL-1 $\beta$ significantly decreased at 40, 80 and $100 \mu \mathrm{g} / \mathrm{mL}$. However, $20 \mu \mathrm{g} / \mathrm{mL}$ açaí
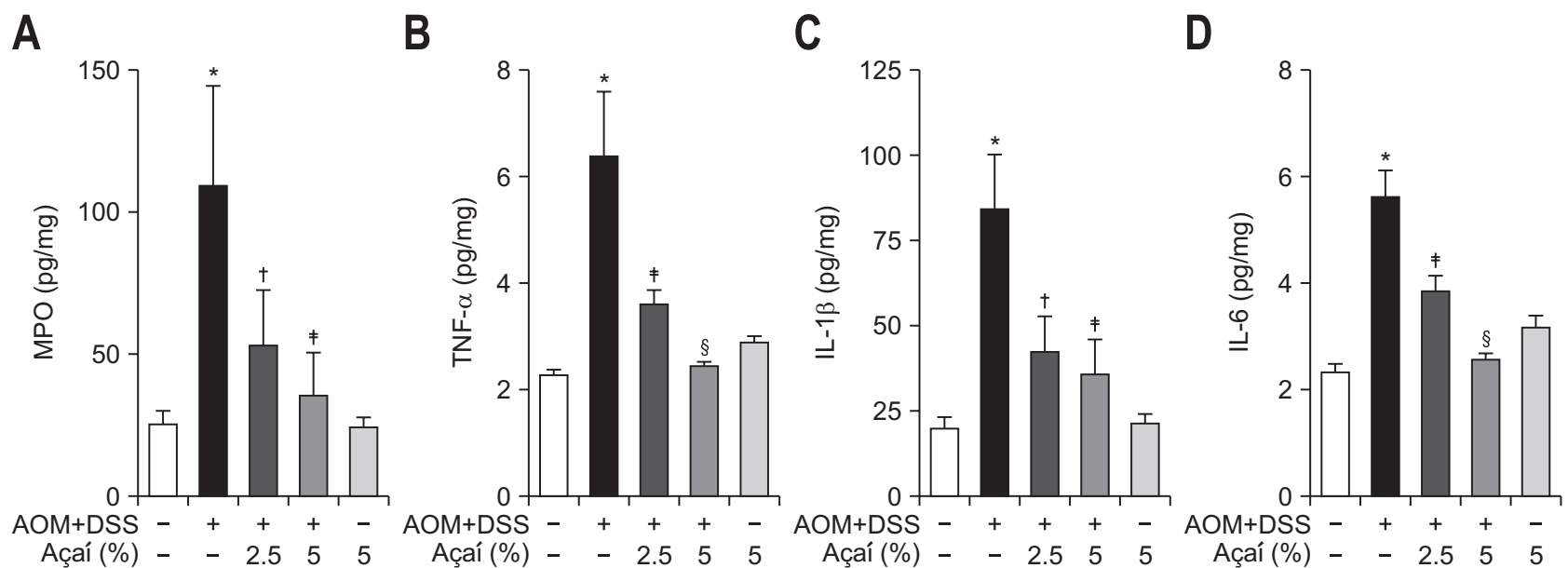

Fig. 3. Açaí downregulates the myeloperoxidase (MPO) and proinflammatory cytokine levels in the mouse colon. (A) MPO, (B) tumor necrosis factor $\alpha$ (TNF- $\alpha$ ), (C) interleukin (IL)-1 $\beta$, and (D) IL-6 levels in the supernatants of colon strips of control mice (n=5), azoxymethane (AOM)/dextran sulfate sodium (DSS)-treated mice $(n=10)$, AOM/DSS plus açai-treated mice $(n=10)$ and açaí-only-treated mice $(n=5)$. The data are expressed as the mean \pm standard errors. ${ }^{*} \mathrm{p}<0.05$ compared with the control; ${ }^{\dagger} \mathrm{p}<0.05,{ }^{\ddagger} \mathrm{p}<0.01,{ }^{\circledR} \mathrm{p}<0.001$ compared with the A0M/DSS-only-treated group. 
A

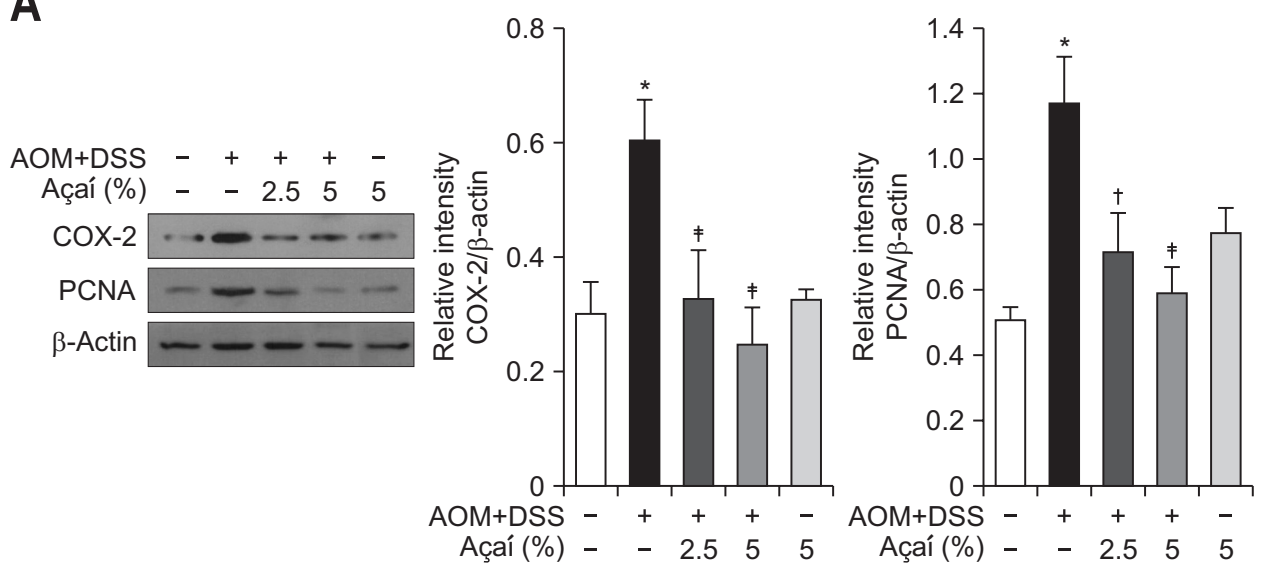

B
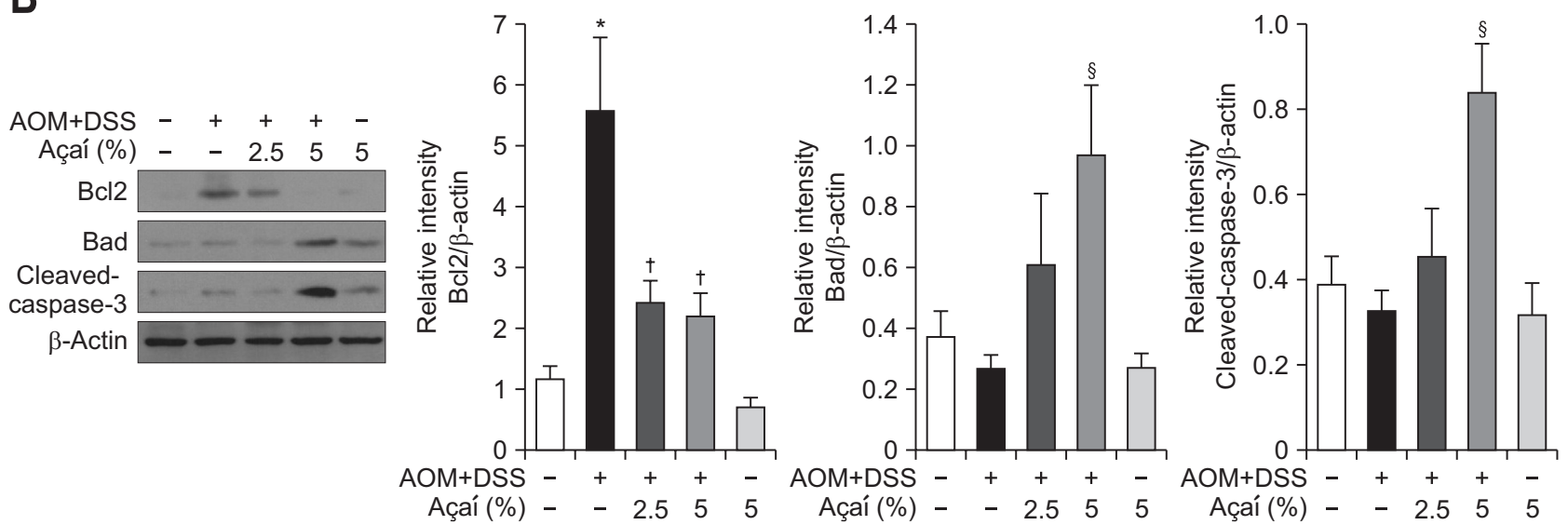

Fig. 4. Açaí inhibits cyclooxygenase $2(\mathrm{COX}-2)$ and proliferating cell nuclear antigen (PCNA) expression and promotes apoptosis. (A) COX-2 and PCNA and (B) B-cell lymphoma 2 (Bcl-2), Bcl-2-associated death promoter (Bad) and cleaved caspase-3 levels in the supernatants of colon strips of control mice $(n=5)$, azoxymethane (AOM)/dextran sulfate sodium (DSS)-treated mice $(n=10), A O M / D S S$ plus açaí-treated mice ( $n=10)$ and acaionly-treated mice $(n=5)$. The data are expressed as the mean \pm standard errors. ${ }^{*} \mathrm{p}<0.05$ compared with the control; ${ }^{\dagger} \mathrm{p}<0.05,{ }^{\ddagger} \mathrm{p}<0.01,{ }^{\S} \mathrm{p}<0.001 \mathrm{com}-$ pared with the AOM/DSS-only-treated group.

extract did not show any significant inhibitory effects.

Regarding cytokine production, RAW 264.7 cells were pretreated with açai extract for 1 hour, stimulated by LPS for 24 hours and TNF- $\alpha$, IL- $1 \beta$ and IL- 6 levels were measured by ELISA. In line with the mRNA findings, those levels were significantly increased upon LPS stimulation (Fig. 5D-F). The TNF- $\alpha$ level was reduced significantly by 20 to $100 \mu \mathrm{g} / \mathrm{mL}$ of açai treatment, while IL-1 $\beta$ was decreased significantly by only 40 and $100 \mu \mathrm{g} / \mathrm{mL}$, and IL- 6 was reduced significantly by 20 and $40 \mu \mathrm{g} / \mathrm{mL}$ of açaí extract. Taken together, these data suggest that açaí may regulate the function of macrophages in terms of proinflammatory cytokine production, thereby ameliorating the development of AOM/DSS-induced CRC.

\section{Açaí upregulates the expression of antioxidant enzymes in CCD841CoN cells}

Açai has a high antioxidant capacity. ${ }^{26}$ Since inflammation often accompanies oxidative stress, we investigated whether açaí could potentiate the antioxidant capacity of intestinal epi- thelial cells in the context of induction of antioxidant enzymes. CCD841CoN human colonic epithelial cells were treated with açaí extract (from 40 to $100 \mu \mathrm{g} / \mathrm{mL}$ ) for 24 hours and then the protein expression of antioxidant enzymes, including HO-1 and NQO-1, were estimated by Western blotting.

Açaí treatment at 40 to $100 \mathrm{\mu g} / \mathrm{mL}$ had no effects on the viability of either RAW 264.7 or CCD841CoN cells (Supplementary Fig. 1). As shown in Fig. 6, açaí treatment (from 40 to $100 \mu \mathrm{g} /$ $\mathrm{mL}$ ) increased the HO-1 protein level in CCD841CoN cells (Fig. 6). The protein level of NQO-1 was also elevated by açai treatment (20 to $100 \mu \mathrm{g} / \mathrm{mL}$ ) (Fig. 6).

\section{DISCUSSION}

In the present study, we investigated the anticarcinogenic effects of açaí using experimental models of inflammationassociated colon carcinogenesis. Açai reduced the incidence of adenoma and cancer and multiplicity of tumors in the AOM/ DSS mouse model. Açaí alleviated the expression of proinflam- 

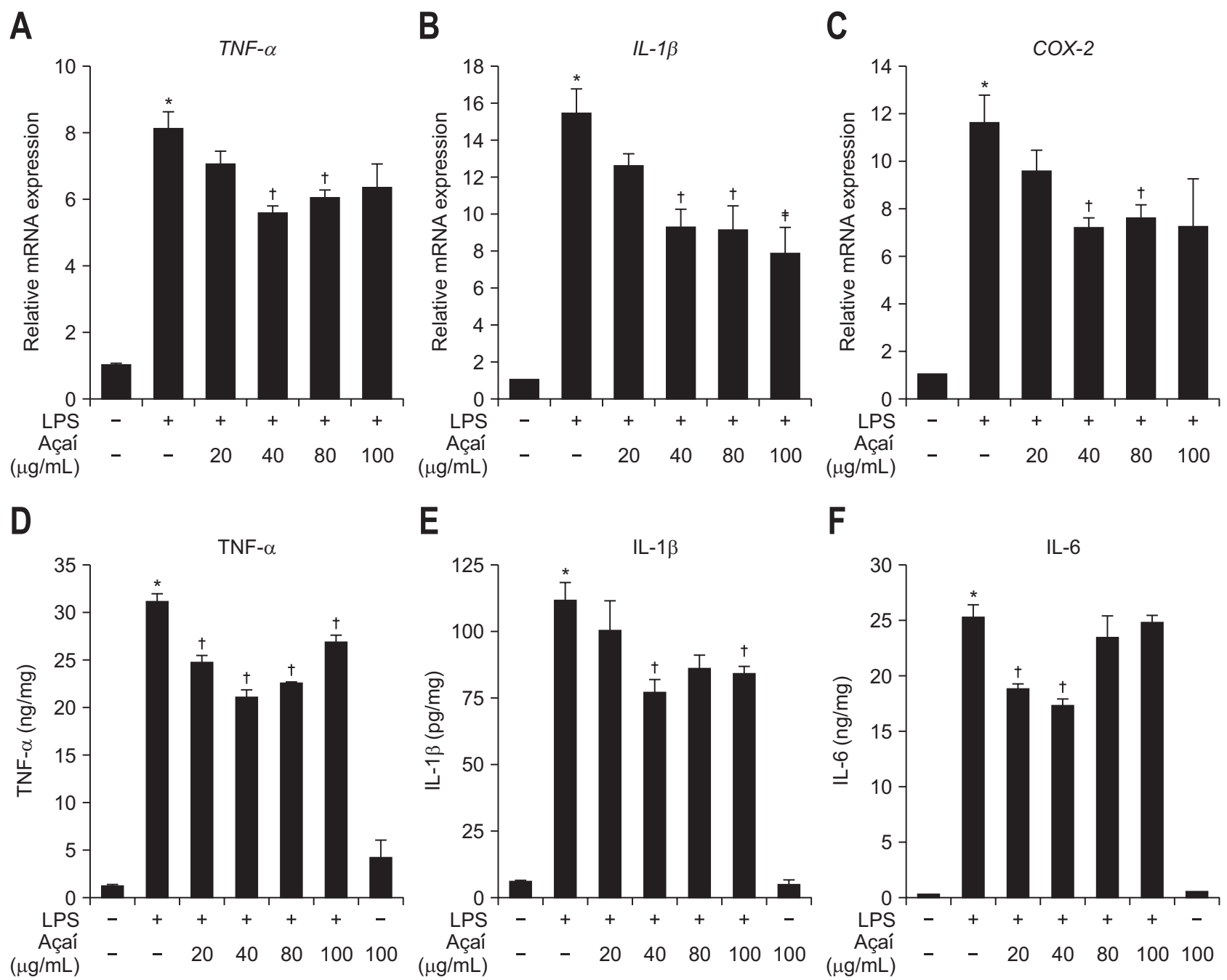

\section{E}

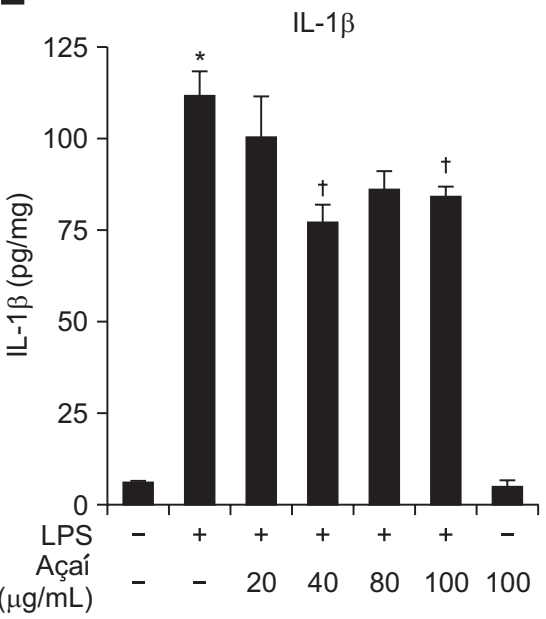

$\mathbf{F}$



Fig. 5. Açaí reduces lipopolysaccharide (LPS)-induced proinflammatory gene expression in RAW 264.7 cells. Reverse transcription-polymerase chain reaction (RT-PCR) analysis of LPS-induced mRNA expression of tumor necrosis factor $\alpha$ (TNF- $\alpha$ ) (A), interleukin (IL)-1 $\beta$ (B) and cyclooxygenase $2(\mathrm{COX}-2)(\mathrm{C})$ and protein levels of TNF- $\alpha(\mathrm{D}), \mathrm{IL}-1 \beta(\mathrm{E})$ and IL-6 (F) in RAW 264.7 cells. The data represent three independent experiments $(n=3)$. The results are expressed as the mean \pm standard errors. ${ }^{*} \mathrm{p}<0.05$ compared with the control; ${ }^{\dagger} \mathrm{p}<0.05$ and ${ }^{\ddagger} \mathrm{p}<0.01$ compared with the LPSonly-treated group.
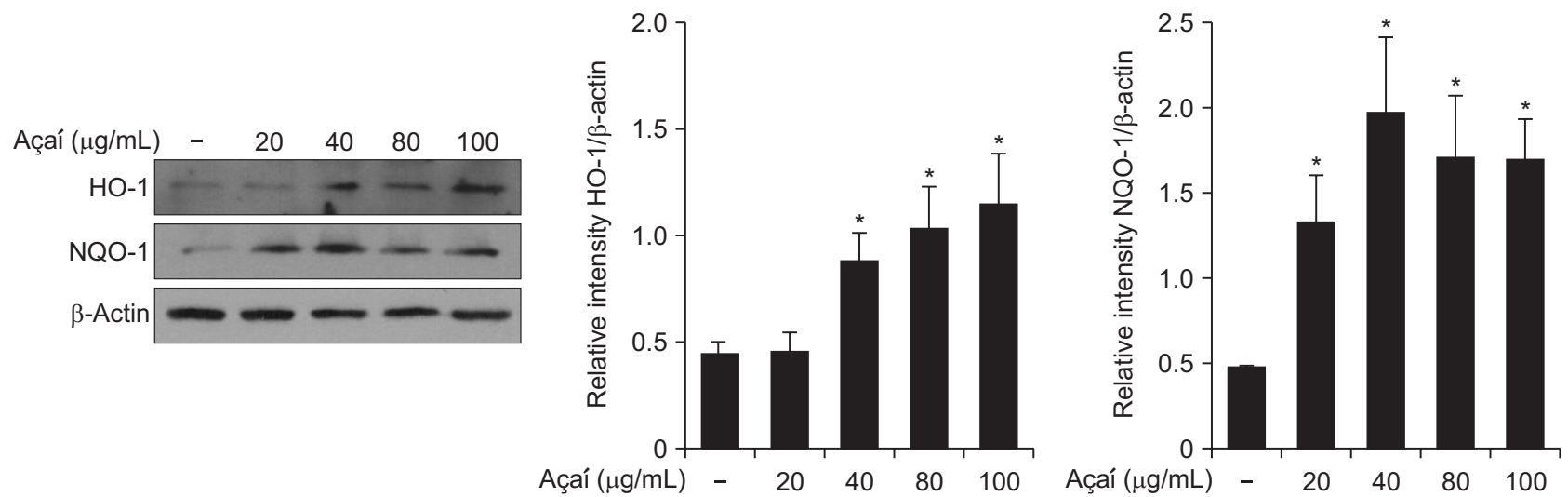

Fig. 6. Açaí induces the expression of antioxidant enzymes in CCD841CoN cells. CCD841CoN cells were treated with the indicated concentrations of açai for 24 hours. Total cellular lysates were collected for the detection of heme oxygenase-1 (HO-1) and NAD(P)H:quinone oxidoreductase 1 (NQO-1) protein expression by Western blot analysis. The data represent three independent experiments $(\mathrm{n}=3)$. The results are expressed as the mean \pm standard errors. ${ }^{*} \mathrm{p}<0.05$ compared with the control. 
matory cytokines and induced apoptosis and production of antioxidant enzymes.

The antitumor effect of açai in the present study is in accordance with the results of Fragoso et al. ${ }^{19}$ In their study, a 5\% açai-containing diet reduced the number of aberrant crypts and cancers in a dimethylhydrazine-induced rat colon carcinogenesis model. However, the mechanism underlying the preventive effect of açai on CRC carcinogenesis was not investigated.

We proceeded to evaluate the mechanism underlying the anti-CRC effect of açaí. First, we demonstrated markedly decreased expressions of IL- $1 \beta$, TNF- $\alpha$, IL- 6 and COX- 2 in LPS-stimulated RAW 264.7 cells and in the mouse colon. Reduced TNF- $\alpha$ expression may be associated with the inactivation of extracellular signal-regulated kinase (ERK), nuclear factor kappa-light-chainenhancer of activated B cells (NF- $\kappa \mathrm{B})$, and phosphatidylinositide 3-kinase and protein kinase B (PI3K-AKT) pathways in epithelial cells, leading to an inhibition of $\beta$-catenin signaling ${ }^{27,28}$ in CRC tumorigenesis.

While inflammation can bypass the mutation requirement for tumor initiation, another important role of inflammation is induction of mutations by persistent ROS production. ${ }^{7}$ The main substances that link inflammation to cancer via oxidative stress are cytokines. ${ }^{29}$ It has been reported that LPS and enhanced proliferation of Gram-negative bacteria in the DSS-treated mouse colon was associated with increased lipid peroxidation..$^{30}$ ROS, in turn, affect the expression of genes that regulate cell differentiation and growth, leading to initiation of cancer. ${ }^{31,32}$ In the present study, açai treatment reduced the levels of inflammatory cytokines in colonic mucosa and macrophages and induced production of antioxidant enzymes in CCD841CoN normal colon epithelial cells. This was consistent with the fact that polyphenolics suppress proliferation of colon cancer cells by reducing ROS levels. ${ }^{33}$ Overall, ROS production may be down-regulated by açai, which could be one of the mechanisms underlying involved the reduced incidence of colonic tumors in this study. Further in vivo study to evaluate the antioxidant property of açaí is warranted.

Finally, antiapoptotic capacity is crucial for survival of cancer cells. Human CRC is associated with increased inhibition of apoptosis, ${ }^{34}$ and mutated colon epithelial cells avoid the normal clearance mechanism, successfully developing to invasive tumors. ${ }^{35,36}$ Dias et al ${ }^{18}$ demonstrated that açaí inhibited growth of SW-480 cells by inducing cytochrome c, cleaved caspase 3, and reducing the level of the antiapoptotic factor poly [ADP-ribose] polymerase 1 (PARP-1). The down-regulation of Bcl-2 by açai in the present study is consistent with previous reports. ${ }^{37,38}$ Marked decreases in the levels of the proliferation factor PCNA were also noted, suggesting that açai suppressed CRC by reducing the antiapoptotic capacity of cancer cells or activating the mitochondrial proapoptotic pathway.

Unfortunately, in the present study, the key mechanisms of prevention against CRC were not demonstrated. It is not clear whether açaí is predominantly anti-inflammatory rather than directly suppressing already established cancer cells, since the viability of colon cancer cells was not inhibited by açaí treatment. Moreover, although the incidence of adenoma and adenocarcinoma were decreased in the 5\% açaí-feeding group, it did not seem that açaí can inhibit cancer submucosal invasion. Therefore, the anti-inflammatory effect or growth inhibition effect of açai may reduce colitis and subsequent CRC or adenoma formation at the early stages. Regarding antioxidant property of açaí, H0-1, NQO-1 and Nrf2 in vivo as well as in vitro should have been measured, but a lack of manpower and funds limited further evaluation. Another limitation of this study is that the açai berry extract used is not thoroughly characterized. Since we used the soluble part of açai powder in this in vitro study, some effective component in the precipitate might be excluded.

Nonetheless, this is one of few studies to demonstrate an anticolon cancer effect of açai in vivo, together with the antiinflammatory and antioxidant effects in vitro. We evaluated the incidence of macroscopically or microscopically detected tumors, not aberrant crypt foci. Although we did not perform a histological evaluation of the entire colon, three independent and experienced colonoscopists identified adenomas in a blinded manner; and lesions about which there was disagreement underwent histological evaluation. Any lesion suspicious for adenocarcinoma, tumors larger than $0.2 \mathrm{~cm}$ in diameter, and the rectum, where tumors occurred most frequently, were also evaluated by an expert pathologist in a blinded manner. Moreover, by dividing tumors into adenoma and adenocarcinoma and assessing the depth of invasion histologically, we aimed to clarify the stage of colon carcinogenesis that was inhibited by açaí.

In conclusion, açai treatment suppressed AOM plus DSSinduced colonic adenoma and cancer formation in mice with no toxicity by reducing COX-2, TNF- $\alpha$, IL- $1 \beta$ and IL- 6 expression levels in macrophages and the mouse colon, suppressing Bcl2 and PCNA, and activating the mitochondrial proapoptotic pathway. Furthermore, açai treatment may protect against ROS production.

Consumption of açai-containing juice exhibited in vivo antiinflammatory and antioxidant properties in human subjects based on a randomized, double-blind, placebo-controlled crossover study. ${ }^{39}$ Further investigations are needed for this formulation to be used against human CRC.

\section{CONFLICTS OF INTEREST}

No potential conflict of interest relevant to this article was reported.

\section{ACKNOWLEDGEMENTS}

The authors are indebted to J. Patrick Barron, Professor Emer- 
itus, Tokyo Medical University and Adjunct Professor, Seoul National University Bundang Hospital for his pro bono editing of this manuscript. In addition, the authors thank the Division of Statistics in the Medical Research Collaborating Center at Seoul National University Bundang Hospital for statistical analyses.

This work was supported by the National Research Foundation of Korea (NRF) grant for the Global Core Research Center (GCRC) funded by the Korea government (MSIP) (No. 20110030001).

\section{REFERENCES}

1. Ferlay J, Shin HR, Bray F, Forman D, Mathers C, Parkin DM. Estimates of worldwide burden of cancer in 2008: GLOBOCAN 2008. Int J Cancer 2010;127:2893-2917.

2. Kuper H, Adami HO, Trichopoulos D. Infections as a major preventable cause of human cancer. J Intern Med 2000;248:171-183.

3. Mantovani A, Allavena P, Sica A, Balkwill F. Cancer-related inflammation. Nature 2008;454:436-444.

4. Feagins LA, Souza RF, Spechler SJ. Carcinogenesis in IBD: potential targets for the prevention of colorectal cancer. Nat Rev Gastroenterol Hepatol 2009;6:297-305.

5. Lakatos PL, Lakatos L. Once daily 5-aminosalicylic acid for the treatment of ulcerative colitis: are we there yet? Pharmacol Res 2008;58:190-195.

6. Meira LB, Bugni JM, Green SL, et al. DNA damage induced by chronic inflammation contributes to colon carcinogenesis in mice. J Clin Invest 2008;118:2516-2525.

7. Grivennikov SI. Inflammation and colorectal cancer: colitis-associated neoplasia. Semin Immunopathol 2013;35:229-244.

8. Becker C, Fantini MC, Schramm C, et al. TGF-beta suppresses tumor progression in colon cancer by inhibition of IL-6 transsignaling. Immunity 2004;21:491-501.

9. Popivanova BK, Kitamura K, Wu Y, et al. Blocking TNF-alpha in mice reduces colorectal carcinogenesis associated with chronic colitis. J Clin Invest 2008;118:560-570.

10. Bollrath J, Phesse TJ, von Burstin VA, et al. gp130-mediated Stat3 activation in enterocytes regulates cell survival and cell-cycle progression during colitis-associated tumorigenesis. Cancer Cell 2009;15:91-102.

11. Grivennikov S, Karin E, Terzic J, et al. IL-6 and Stat3 are required for survival of intestinal epithelial cells and development of colitisassociated cancer. Cancer Cell 2009;15:103-113.

12. Half E, Arber N. Colon cancer: preventive agents and the present status of chemoprevention. Expert Opin Pharmacother 2009;10:211219.

13. Das D, Arber N, Jankowski JA. Chemoprevention of colorectal cancer. Digestion 2007;76:51-67.

14. Poulose SM, Fisher DR, Larson J, et al. Anthocyanin-rich açai (Euterpe oleracea Mart.) fruit pulp fractions attenuate inflammatory stress signaling in mouse brain BV-2 microglial cells. J Agric Food Chem 2012;60:1084-1093.
15. Xie C, Kang J, Burris R, et al. Açaí juice attenuates atherosclerosis in ApoE deficient mice through antioxidant and anti-inflammatory activities. Atherosclerosis 2011;216:327-333.

16. Moura RS, Ferreira TS, Lopes AA, et al. Effects of Euterpe oleracea Mart. (AÇAÍ) extract in acute lung inflammation induced by cigarette smoke in the mouse. Phytomedicine 2012;19:262-269.

17. Del Pozo-Insfran D, Percival SS, Talcott ST. Açai (Euterpe oleracea Mart.) polyphenolics in their glycoside and aglycone forms induce apoptosis of HL-60 leukemia cells. J Agric Food Chem 2006;54:1222-1229.

18. Dias MM, Noratto G, Martino HS, et al. Pro-apoptotic activities of polyphenolics from açai (Euterpe oleracea Martius) in human SW480 colon cancer cells. Nutr Cancer 2014;66:1394-1405.

19. Fragoso MF, Romualdo GR, Ribeiro DA, Barbisan LF. Açai (Euterpe oleracea Mart.) feeding attenuates dimethylhydrazine-induced rat colon carcinogenesis. Food Chem Toxicol 2013;58:68-76.

20. Stoner GD, Wang LS, Seguin C, et al. Multiple berry types prevent $\mathrm{N}$-nitrosomethylbenzylamine-induced esophageal cancer in rats. Pharm Res 2010;27:1138-1145.

21. National Research Council (US) Subcommittee on Laboratory Animal Nutrition. Nutrient requirements of laboratory animals, fourth ed. Washington, DC: National Academy Press, 1995.

22. Yum HW, Zhong X, Park J, et al. Oligonol inhibits dextran sulfate sodium-induced colitis and colonic adenoma formation in mice. Antioxid Redox Signal 2013;19:102-114.

23. Hamilton SR, Aaltonen LA. World Health Organization classification of tumors: pathology and genetics of tumours of the digestive system. Lyon: IARC Press, 2000.

24. Surh YJ, Chun KS, Cha HH, et al. Molecular mechanisms underlying chemopreventive activities of anti-inflammatory phytochemicals: down-regulation of COX-2 and iNOS through suppression of NF-kappa B activation. Mutat Res 2001;480-481:243-268.

25. Kubben FJ, Peeters-Haesevoets A, Engels LG, et al. Proliferating cell nuclear antigen (PCNA): a new marker to study human colonic cell proliferation. Gut 1994;35:530-535.

26. Mertens-Talcott SU, Rios J, Jilma-Stohlawetz P, et al. Pharmacokinetics of anthocyanins and antioxidant effects after the consumption of anthocyanin-rich acai juice and pulp (Euterpe oleracea Mart.) in human healthy volunteers. J Agric Food Chem 2008;56:7796-7802.

27. Tessner TG, Muhale F, Riehl TE, Anant S, Stenson WF. Prostaglandin E2 reduces radiation-induced epithelial apoptosis through a mechanism involving AKT activation and bax translocation. J Clin Invest 2004;114:1676-1685.

28. Pozzi A, Yan X, Macias-Perez I, et al. Colon carcinoma cell growth is associated with prostaglandin E2/EP4 receptor-evoked ERK activation. J Biol Chem 2004;279:29797-29804.

29. Federico A, Morgillo F, Tuccillo C, Ciardiello F, Loguercio C. Chronic inflammation and oxidative stress in human carcinogenesis. Int J Cancer 2007;121:2381-2386.

30. Lee IA, Bae EA, Hyun YJ, Kim DH. Dextran sulfate sodium and 2,4,6-trinitrobenzene sulfonic acid induce lipid peroxidation by 
the proliferation of intestinal gram-negative bacteria in mice. J Inflamm (Lond) 2010;7:7.

31. Schumacker PT. Reactive oxygen species in cancer cells: live by the sword, die by the sword. Cancer Cell 2006;10:175-176.

32. Petros JA, Baumann AK, Ruiz-Pesini E, et al. mtDNA mutations increase tumorigenicity in prostate cancer. Proc Natl Acad Sci U S A 2005;102:719-724.

33. Gupta SC, Hevia D, Patchva S, Park B, Koh W, Aggarwal BB. Upsides and downsides of reactive oxygen species for cancer: the roles of reactive oxygen species in tumorigenesis, prevention, and therapy. Antioxid Redox Signal 2012;16:1295-1322.

34. Watson AJ. Apoptosis and colorectal cancer. Gut 2004;53:17011709.

35. Kim WK, Bang MH, Kim ES, et al. Quercetin decreases the expression of ErbB2 and ErbB3 proteins in HT-29 human colon cancer cells. J Nutr Biochem 2005;16:155-162.
36. Athar M, Back JH, Kopelovich L, Bickers DR, Kim AL. Multiple molecular targets of resveratrol: anti-carcinogenic mechanisms. Arch Biochem Biophys 2009;486:95-102.

37. Ramos S. Cancer chemoprevention and chemotherapy: dietary polyphenols and signalling pathways. Mol Nutr Food Res 2008;52:507526.

38. Noratto GD, Jutooru I, Safe S, Angel-Morales G, Mertens-Talcott SU. The drug resistance suppression induced by curcuminoids in colon cancer SW-480 cells is mediated by reactive oxygen species-induced disruption of the microRNA-27a-ZBTB10-Sp axis. Mol Nutr Food Res 2013;57:1638-1648.

39. Jensen GS, Wu X, Patterson KM, et al. In vitro and in vivo antioxidant and anti-inflammatory capacities of an antioxidantrich fruit and berry juice blend: results of a pilot and randomized, double-blinded, placebo-controlled, crossover study. J Agric Food Chem 2008;56:8326-8333. 